\title{
Drainage of single Plateau borders: Direct observation of rigid and mobile interfaces
}

\author{
Stephan A. Koehler, ${ }^{1,3}$ Sascha Hilgenfeldt, ${ }^{2}$ Eric R. Weeks, ${ }^{3}$ and Howard A. Stone ${ }^{1}$ \\ ${ }^{1}$ Division of Engineering and Applied Sciences, Pierce Hall, Harvard University, Cambridge, Massachusetts 02138 \\ ${ }^{2}$ Faculty of Applied Physics, University of Twente, P.O. Box 217, 7500AE Enschede, The Netherlands \\ ${ }^{3}$ Department of Physics, Emory University, Atlanta, Georgia 30322
}

(Received 23 December 2001; published 9 October 2002)

\begin{abstract}
Foam drainage varies with surfactant. We present direct measurements of the flow velocity profiles across single Plateau borders, which make up the interconnected channel-like network for liquid flow. For protein foams the interface is rigid, whereas small-surfactant foams show significant interfacial mobility. The results agree with a model that takes into account the shearing of the liquid-gas interface transverse to the flow direction. A significant consequence is that bubble size and liquid volume fraction in a foam affect the relative importance of surface rheology on the drainage behavior.
\end{abstract}

DOI: 10.1103/PhysRevE.66.040601

PACS number(s): 68.03. $-\mathrm{g}$, 47.55.Mh, 82.70.Rr, 82.70.Uv

Foams and emulsions are common complex soft materials with many applications. Their macroscopic properties are determined by the volume fraction of the dispersed phase, the material properties of the continuous and dispersed phases, and the surfactants used to stabilize the system. One important physical process in foams is the flow of liquid in the network of interstitial spaces (channels) between the bubbles, referred to as foam drainage. Macroscopic measurements of foam drainage from different laboratories have yielded discrepant results, which have been attributed to changes in the microscopic flow field, likely due to differences in surface rheology between the surfactants used [1-6]. Surfactant-related effects such as surface viscosity, Marangoni stresses, or marginal regeneration are known to influence the drainage of isolated soap films [7,8]. In this paper we report the direct observations of the velocity field across foam liquid channels (Plateau borders) and find significant differences depending on the surfactant type. The results of this study provide a rational path for connecting microscopic flows to macroscopic foam drainage measurements.

Foams are often dry, i.e., little liquid resides between the bubbles, and very little liquid is found in the thin films that separate adjacent bubbles. Most of the liquid is in Plateau borders, the region between three adjacent bubbles. The Plateau borders are slender and straight, with a scallopedtriangle cross section [see Figs. 1(a) and 2]. A macroscopic, effective-medium description of foam drainage (i.e., on a scale much larger than the bubble size) is an average over the microscopic flow through single Plateau borders and their junctions. Thus, the assumptions of microscopic modeling dictate the predictions of foam drainage theories. For instance, large, insoluble surfactant molecules are expected to form rigid interfacial layers, enforcing zero-velocity boundary conditions for the flow through the Plateau borders. Small, soluble surfactant molecules likely yield significant interfacial mobility and faster drainage [3,4,9].

In the pioneering paper by Leonard and Lemlich [1], a model for drainage through foams is presented based upon flow through an individual (infinite) Plateau border (see also Ref. [10]). The influence of surface rheology is included through surface viscosity effecting resistance from transverse shear along the interfaces of the Plateau borders. The coupling of bulk and surface flows is modeled by three parameters: the Plateau border width (and radius of curvature) $a$, the shear viscosity of the bulk fluid, $\mu$, and the surface shear viscosity, $\mu_{s}$. The dimensionless ratio $M \equiv \mu a / \mu_{s}$ (the inverse of the Boussinesq number [11]) determines the mobility of the channel surface.

Attributing macroscopic drainage behavior to a microscopic property such as interfacial mobility is an indirect way of reasoning, and thus somewhat unsatisfactory, despite several supporting experiments [3,9]. We therefore set up an experiment for microscopic drainage measurements. A Noran confocal microscope tilted on its side imaged the flow field inside a single Plateau border of the foam. The foaming solution was seeded with $1-\mu \mathrm{m}$-diameter fluorescent latex spheres (volume fraction $\sim 10^{-6}$ ) [12]. The particle-laden fluid was injected into the foam a few centimeters above the microscope's field of view, thus creating a forced drainage experiment [13]. The advantage of the confocal microscope is its ability to image narrow slices; here we primarily used a $5 \times$ lens, which resulted in imaging slice thicknesses of $\approx 10 \mu \mathrm{m}$ at working distances of about $5 \mathrm{~mm}$; see Fig. 1(a).
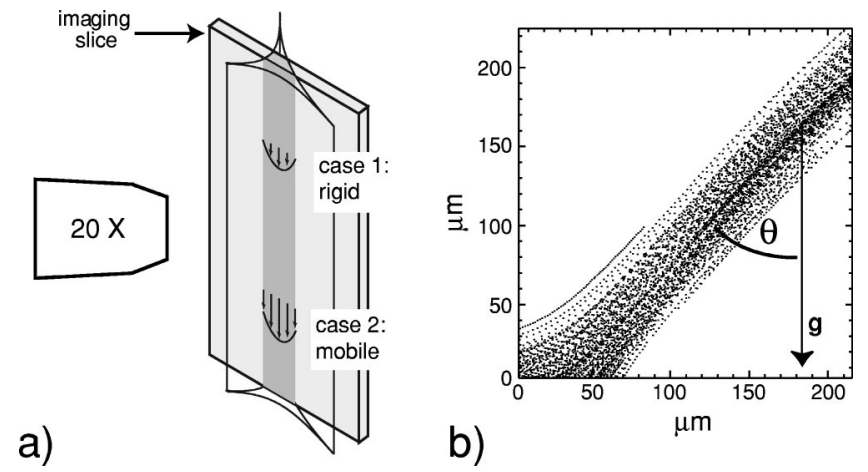

FIG. 1. (a) Schematic of a Plateau border imaged in a confocal slice. Velocity profiles across the Plateau border are sketched for rigid and mobile interfaces. (b) Composite picture of experimental microsphere flow paths through the Plateau border of a SDS foam; $\theta$ is the channel's angle relative to the vertical. 

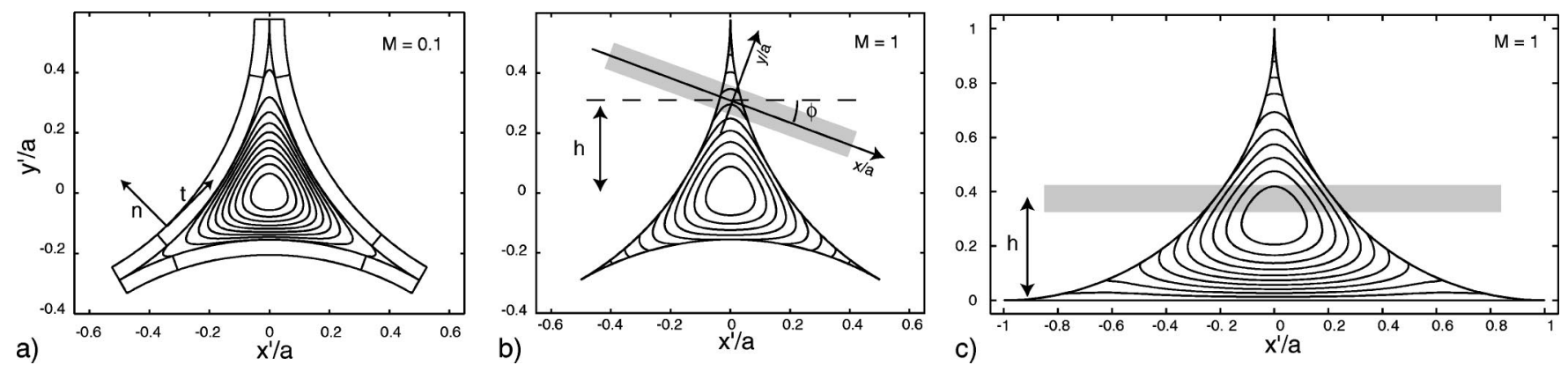

FIG. 2. Velocity contour plots of axial flow (out of the page) through a cross section of interior Plateau borders with mobility $M=0.1$ (a) and $M=1$ (b), and an exterior Plateau border in (c), plotted in coordinates relative to the channel, $x^{\prime} / a, y^{\prime} a$. For ease of presentation, the surface layer thickness shown in (a) is exaggerated 100 times. Ten evenly spaced contour lines are shown, and the maximum velocity $u_{\text {max }}$ is given in terms of the velocity scale $U=a^{2} \rho g \cos \theta / \mu$. In plot (b) the shaded box indicates a confocal imaging slice centered at $h$ $=0.3 a$ (distance from origin), with tilt angle $\phi=-20^{\circ}$, and the coordinate system of the images $x / a, y / a$. Part (c) shows an imaging slice at $h=0.4 a$ from the bottom.

A slow flow of air through a syringe needle submerged in the foaming solution filled a cylindrical tube with monodisperse bubbles of channel length $L \approx 1-2 \mathrm{~mm}$. The tube was notched to accommodate a cover slip for imaging with the confocal microscope. Two different surfactants were used: a protein solution composed of bovine serum albumin (BSA) and the cosurfactant propylene glycol alginate $(\approx 4 \mathrm{~g} / \mathrm{l}$ each) in a $p \mathrm{H} 4.0$ buffer solution, and a soap solution composed of sodium dodecyl sulfate $(\mathrm{SDS})(\approx 1 \mathrm{~g} / \mathrm{l})$ in distilled water. The bulk viscosities are $\mu \approx 0.07 \mathrm{~g} / \mathrm{cm} / \mathrm{s}$ and $\approx 0.01 \mathrm{~g} / \mathrm{cm} / \mathrm{s}$ for the protein solution and SDS, respectively, as measured with a Couette rheometer [14].

The vertical foam tube was attached to the translating stage of the microscope; in the imaging region a cover slip replaced the tube wall. Movies at the rates of 30 or 120 frames/s were recorded for 10-30 s. Particle-tracking software was used to determine the positions and velocities of the microspheres; particles appearing out of focus, too large, or too small, were rejected [15]. Typical flow velocities were below $1000 \mu \mathrm{m} / \mathrm{s}$. Figure 1(b) shows a superposition of all the $\approx 1000$ tracked particles at all times of a movie. The measured flow fields did not vary significantly along the length of the Plateau border or in time. We image both exterior channels i.e., those running along the cover slip, see Fig. 2(c) and interior channels [a few bubble diameters away from the container wall, see Figs. 2(a,b)]. Interior channel imaging requires that the optical path is minimally disturbed by intermediate channels, nodes or films. Therefore, our measurements were performed at liquid volume fractions $\lesssim 10^{-3}$, much lower than in most macroscopic experiments.

The velocity profiles in Figs. 3 and 4 are representative of the roughly 20 successful profiles taken. The width of the channel is divided into $n$ equally spaced bins, for which an average velocity (open circles) is determined by particle tracking. The measurements show that the protein foam has essentially rigid surfaces, whereas the SDS foam shows significant surface velocities. These results agree qualitatively with what has been reported for isolated soap films $[5,7,8]$. Although films in SDS foams are mobile as well, at low volume fractions they are very thin and are not expected to contribute significantly to the overall liquid drainage.
We model the flow through a Plateau border using Stokes's equation with a boundary condition accounting for surface viscosity. For axial flow down an infinite, straight Plateau border there are no longitudinal pressure gradients, and we obtain $\mu \nabla^{2} u+\rho g \cos \theta=0$, where $\mu$ is the (bulk) shear viscosity, $u$ is the axial liquid velocity, $g$ the gravitational acceleration, and $\theta$ the angle of the Plateau border with respect to the vertical [Fig. 1(b)].

At the liquid-gas interface of the channel, the bulk flow shears the surface, and induces a surface flow. At the three "corners," where the Plateau border merges into the films, the liquid velocity is set to zero (see Fig. 2). This pinning is justified by detailed observations of flow in individual films in a foam [1]. In particular, we observe that liquid in mobile films often flows upwards close to a Plateau border, so that the velocity must go to zero near the corner. For exterior Plateau borders, the velocity is also set to zero along the cover slip [Fig. 2(c)]. All other parts of the surfaces are free to move according to the tangential stress balance invoking transverse shear, $\mu_{s} \partial_{t}^{2} u-\mu \partial_{n} u=0$, where $\partial_{t}$ and $\partial_{n}$ are the directional derivatives tangential and normal to the interface, respectively [sketched in Fig. 2(a)]. The dimensionless parameter $M=\mu a / \mu_{s}$ sets the mobility of the interface [16].

For small $M$ the simulation results in an essentially rigid surface with the contour lines tangential to it [see Fig. 2(a)]. The surface mobility increases with $M$ [Fig. 2(b)]. In the limit of zero surface viscosity $(M \rightarrow \infty)$ the contour lines are normal to the interface. An exterior Plateau border is simulated in Fig. 2(c), where the bottom is touching the container wall.

Comparing the measured velocity profiles with simulations requires both geometrical knowledge of the channel and imaging slice as well as knowledge of material parameters such as bulk and surface viscosities. Direct measurements give $\rho, \mu$, and $\theta$. The channel width $a$, tilt angle $\phi$, and location of the imaging slice $h / a$ are unknown. As the reported surface viscosities of SDS vary widely from $\mu_{s}$ $\approx 10^{-5}$ to $3 \times 10^{-3} \mathrm{~g} / \mathrm{s}[7,17-19]$, we also treat $\mu_{s}$ as a free parameter. Therefore we attempt fits for the velocity profiles varying $a, M, \phi$, and $h / a$ in order to test the validity of the simple model originally proposed by Leonard and Lemlich [1]. 

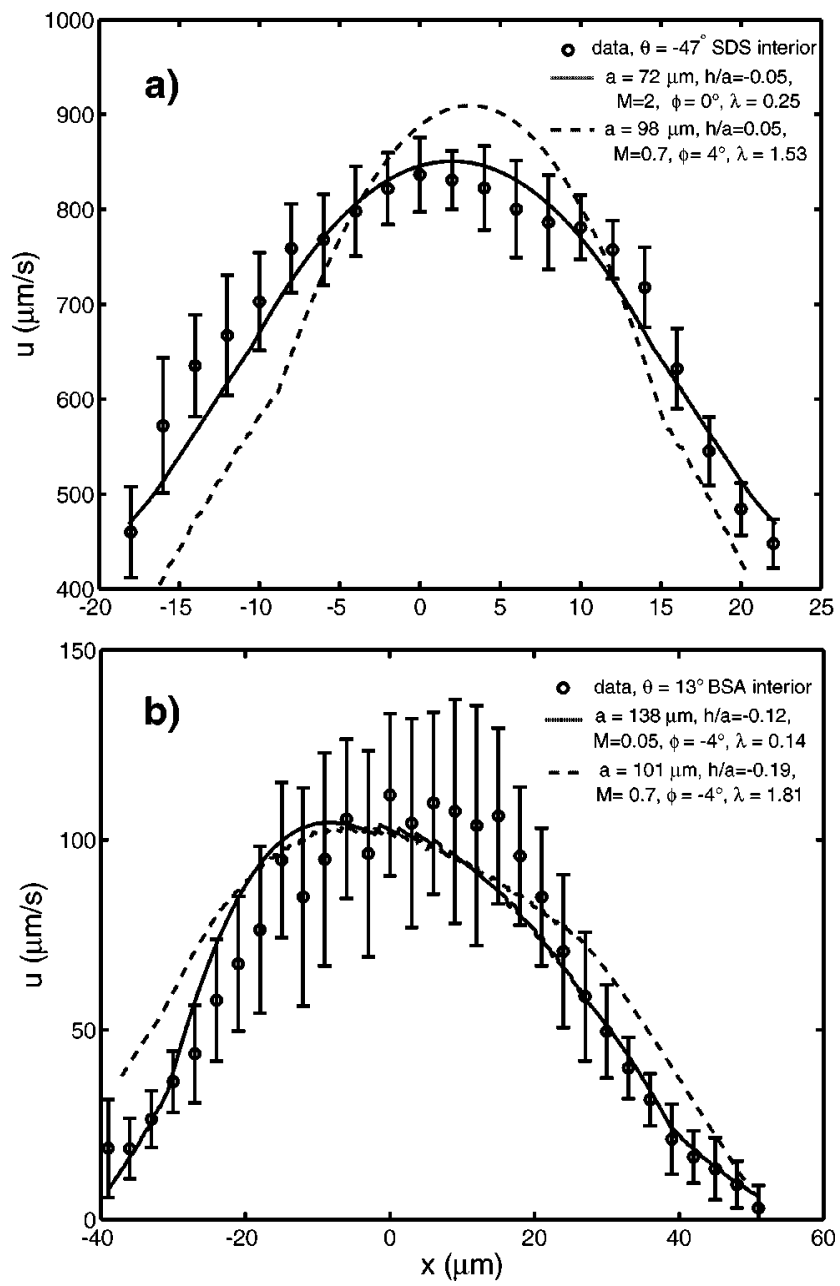

FIG. 3. Measured velocity profiles (circles) and simulations (curves) of interior Plateau borders for (a) SDS and (b) protein foams. For SDS, the best fit (solid line) gives fitting parameters shown in the legend, and a surface viscosity of $\mu_{s} \approx 3.6$ $\times 10^{-5} \mathrm{~g} / \mathrm{s}$. A marginal fit (dashed line) with $\mu_{s} \approx 1.4 \times 10^{-4} \mathrm{~g} / \mathrm{s}$ increases $\lambda$ and can be rejected with $75 \%$ confidence. For BSA, the best fit (solid line) yields $\mu_{s} \approx 1.9 \times 10^{-2} \mathrm{~g} / \mathrm{s}$, while a more mobile interface (dashed line) with $\mu_{s} \approx 1.0 \times 10^{-3} \mathrm{~g} / \mathrm{s}$ yields a worse fit which can be rejected with $80 \%$ confidence.

In Figs. 3 and 4 we compare the experimental data to simulated profiles for different $M$, with $a, \phi, h / a$ adjusted for a best fit. The quality of the fits is determined as follows: Let $\left(x_{i}^{\mathrm{ex}}, u_{i}^{\mathrm{ex}}\right)$ be the position and velocity of the $n$ bins, with standard deviations $\sigma_{x_{i}}$ and $\sigma_{u_{i}}$. For $\sigma_{x_{i}}$ we take one-half of the bin width. For a given choice of fit parameters, the simulated profile is evaluated at $n$ equidistant points located at $\left(x_{i}^{\text {num }}, u_{i}^{\text {num }}\right)$. We then perform a $\chi^{2}$ test for the quantity

$$
\lambda \equiv \frac{1}{2 n} \sum_{i=1}^{n}\left(\frac{u_{i}^{\mathrm{ex}}-u_{i}^{\mathrm{num}}}{\sigma_{u_{i}}}\right)^{2}+\left(\frac{x_{i}^{\mathrm{ex}}-x_{i}^{\mathrm{num}}}{\sigma_{x_{i}}}\right)^{2} .
$$

For interior Plateau borders with the protein surfactant [Fig. 3(b)], the velocities at the interface are small, and the best fits yield small surface mobilities, $M \ll 1$, which are essentially indistinguishable from fits assuming rigid interfaces
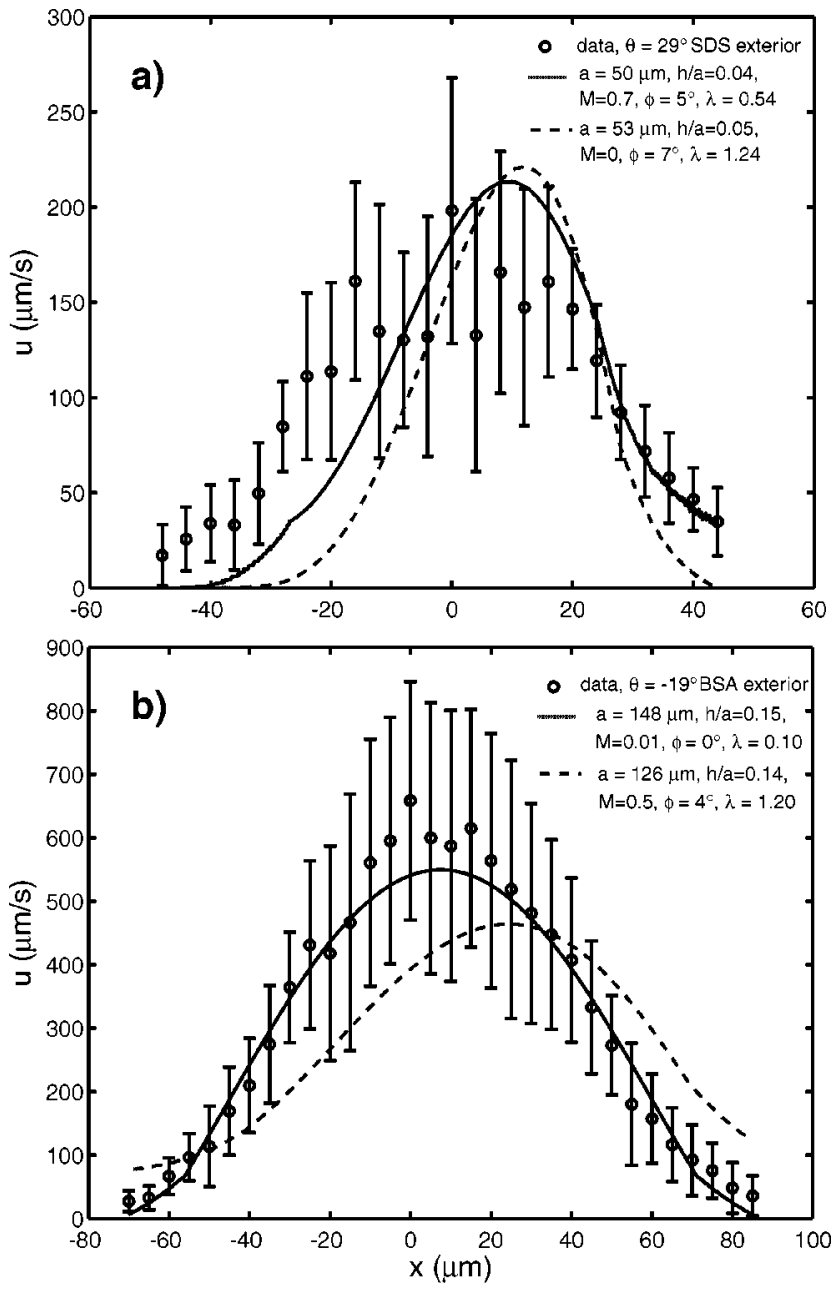

FIG. 4. Velocity profiles of exterior Plateau borders. For SDS (a) the best fit (solid line) gives a surface viscosity of $\mu_{s} \approx 7.1$ $\times 10^{-5} \mathrm{~g} / \mathrm{s}$, whereas a marginal fit (dashed line) with rigid interfaces $\left(\mu_{s}=\infty\right)$ can be rejected with $60 \%$ confidence. For protein (b) the best fit (solid line) gives a surface viscosity of $\mu_{s} \approx 0.10 \mathrm{~g} / \mathrm{s}$, and a marginal fit (dashed line) with $\mu_{s}=1.8 \times 10^{-3} \mathrm{~g} / \mathrm{s}$ can be rejected with $70 \%$ confidence.

(i.e., $\left.\mu_{s}=\infty\right)$. Marginal fits using $\mu_{s} \gtrsim 2.2 \times 10^{-3} \mathrm{~g} / \mathrm{s}$ can be rejected with a confidence $\gtrsim 68 \%$. SDS profiles [Fig. 3(a)], on the other hand, show significant surface mobility. We find $\mu_{s} \approx 3.6 \times 10^{-5} \mathrm{~g} / \mathrm{s}$ as a best-fit value. Attempting to fit the SDS velocity profile with $M=0$ yields $\lambda \gg 10$, so the rigidinterface hypothesis can be rejected with $>99 \%$ confidence.

The differences between exterior Plateau border profiles of soap and protein foams (Fig. 4) are less pronounced. The rigid wall severely restricts the flow velocity over the whole channel regardless of the interfacial mobility. The measurements are consistent with a wider range of $\mu_{s}$, and the rigidinterface hypothesis for SDS can be rejected with $60 \%$ confidence.

Because of the finite thickness of the imaging slice, the velocity profile is an average over a range of velocity profiles of different widths from different locations in the channel. This averaging distorts the edges of the velocity profile from 
a parabolic shape, adding "wings," which are reproduced in the simulations (Figs. 3 and 4).

We have measured velocity profiles in individual Plateau borders of aqueous foams, and shown the influence of surfactants on the interfacial boundary condition. Protein surfactants result in essentially zero surface velocities, whereas for SDS the surface velocities are high, resulting in high bulk velocities. A model including interfacial shear transverse to the flow direction, based upon ideas from Ref. [1] agrees with experiments and is consistent with reported values for the surface viscosity.

During foam drainage, the gravitational force is balanced by a combination of Marangoni and viscous forces from the bulk and the surface. Durand and Langevin consider the influence of Marangoni stresses in a model with simplified cylindrical geometry that neglects transverse variations, and find very small interfacial velocities [20]. However, in a slender Plateau border, the transverse shear contribution as modeled in the present work becomes significant. Recent work on macroscopic drainage experiments by Carrier and Colin
[21] also stress the importance of transverse viscous surface shear.

The mobility parameter $M$ quantifies the ratio of bulk and surface dissipation and so determines macroscopic drainage rates. As the mobility depends on the channel width ( $M$ $\propto a)$, which increases with both the liquid volume fraction and bubble size $L\left(a \propto L \epsilon^{1 / 2}\right)$, the drainage behavior is determined by both its geometry and its surface chemistry. Thus we predict that, for a given surfactant, a foam with very small bubbles should behave according to the rigid-channel model $[22,23]$. However, large bubbles could have mobile liquid-gas interfaces, and the dominant dissipation should occur in the nodes, so that "node-dominated" foam drainage [4] is a better approximation.

We thank J. Krocak and D. Weitz for their help with the confocal setup. The Harvard MRSEC and the Petroleum Research Fund (Grant No. 35926-AC9) are thanked for their support, as is the Netherlands Organization for Scientific Research (NWO).
[1] R.A. Leonard and R. Lemlich, AIChE J. 11, 18 (1965).

[2] Z. Zapryanov, A.K. Malhotra, N. Aderangi, and D.T. Wasan, Int. J. Multiphase Flow 9, 105 (1983).

[3] M. Durand, G. Martinoty, and D. Langevin, Phys. Rev. E 60, R6307 (1999).

[4] S.A. Koehler, S. Hilgenfeldt, and H.A. Stone, Phys. Rev. Lett. 82, 4232 (1999).

[5] P.J. Wilde, A.R. Mackie, F.A. Husband, A.P. Gunning, V.J. Morris, and A. Fillery-Travis, in Proceedings of the International Workshop on Foams and Films, Leuven, Belgium, edited by D. Weaire and J. Banhart (MIT-Verlag, Bremen, 1999), p. 59.

[6] S. Hutzler and D. Weaire, Philos. Mag. Lett. 80, 419 (2000).

[7] K.J. Mysels, K. Shinoda, and S. Frankel, Soap Films, Studies of their Thinning (Pergamon Press, London, 1959).

[8] P.J.M. Baets and H.N. Stein, Langmuir 8, 2199 (1992).

[9] S.A. Koehler, S. Hilgenfeldt, and H.A. Stone, Langmuir 16, 6327 (2000).

[10] D. Desai and R. Kumar, Chem. Eng. Sci. 37, 1361 (1982).

[11] D.A. Edwards, H. Brenner, and D.T. Wasan, Interfacial Transport Processes and Rheology (Butterworth-Heinemann, Boston, 1991).

[12] The microspheres were left in the surfactant solution, so any possible interactions with the surfactants would have occurred before the actual foam drainage experiments, and we do not expect any particle-interface interactions.
[13] G. Verbist, D. Weaire, and A. Kraynik, J. Phys.: Condens. Matter 8, 3715 (1996).

[14] The protein solution shows slightly non-Newtonian behavior. The given value of $\mu$ for shear rates is comparable to those in the experiments described here.

[15] A.D. Dinsmore, E.R. Weeks, V. Prasad, A.C. Levitt, and D.A. Weitz, Appl. Opt. 40, 4152 (2001).

[16] The surface stress boundary condition is conveniently implemented numerically by treating the surface layer not as a twodimensional object, but as a liquid layer of finite thickness $d$ and bulk viscosity $\mu^{*}$, such that $\mu_{s}=d \mu^{*}$, see P.G. Saffman, J. Fluid Mech. 73, 593 (1976). Numerical solutions converge to a well-defined limit as $d / a \rightarrow 0$. In practice, $d / a=5 \times 10^{-4}$ was found sufficient to reach this limit.

[17] D.O. Shah, N.F. Djabbarah, and D.T. Wasan, Colloid Polym. Sci. 256, 1002 (1978).

[18] J.T. Petkov, K.D. Danov, N.D. Denkov, R. Aust, and F. Durst, Langmuir 12, 2650 (1996).

[19] C. Barentin, C. Ybert, J.M. di Meglio, and J.F. Joanny, J. Fluid Mech. 397, 331 (1999).

[20] M. Durand and D. Langevin, Eur. Phys. J. E 7, 35 (2002).

[21] V. Carrier and A. Colin (private communication).

[22] I.I. Gol'dfarb, K.B. Kann, and I.R. Shreiber, Fluid Dyn. 23, 244 (1988).

[23] D. Weaire, N. Pittet, S. Hutzler, and D. Pardal, Phys. Rev. Lett. 71, 2670 (1993). 